\section{The 2016 Clinical Practice Guidelines for Management of Hospital-Acquired and Ventilator-Associated Pneumonia}

Hospital-acquired pneumonia and ventilator-associated pneumonia are defined as occurring at least $48 \mathrm{~h}$ after admission or endotracheal intubation, respectively. ${ }^{1}$ Until recently, evidence-based guidelines for treating these illnesses were quite dated. The last edition of the guideline prepared by the American Thoracic Society (ATS) and the Infectious Diseases Society of America (IDSA) was published in 2005, ${ }^{2}$ and the latest Canadian national guidelines, prepared by the Association of Medical Microbiology and Infectious Disease Canada (AMMI Canada), were published in 2008. ${ }^{3}$ The 2016 IDSA/ATS update ${ }^{1}$ was therefore much-awaited, and it includes several significant changes to recommendations from the previous edition.

For pharmacists, perhaps the most relevant changes are those concerning recommended empiric antimicrobial regimens. Determination of risk factors for multidrug-resistant (MDR) pathogens continues to be promoted for therapeutic decisionmaking. ${ }^{1}$ However, the factors to consider have been modified for greater precision. For example, because of inconsistent data linking the timing of pneumonia onset with MDR pathogens, the distinction between early- and late-onset hospital-acquired pneumonia has been removed. ${ }^{1}$ Although the 2016 update continues to list " 5 or more days of hospitalization preceding pneumonia occurrence" as a risk factor for MDR pathogens, this now applies to ventilator-associated pneumonia only and is superseded by the presence of other risk factors. ${ }^{1}$ On a related note, the concept of health care-associated pneumonia has been removed entirely, with an expectation that the risk factors for MDR pathogens associated with this previously recognized entity will be refined and included in forthcoming guidelines for community-acquired pneumonia. ${ }^{1}$

Importantly, despite greater nuance in identifying the risk of MDR pathogens, recommended empiric antimicrobial regimens are now more uniformly broad-spectrum. For example, at least one antipseudomonal agent is now recommended for both hospital-acquired and ventilator-associated pneumonia, regardless of patient-specific risk factors. ${ }^{1}$ This new recommendation contrasts with the 2005 edition, ${ }^{2}$ which listed regimens with no pseudomonal coverage (e.g., ceftriaxone or moxifloxacin monotherapy) as alternatives for early-onset hospital-acquired or ventilator-associated pneumonia in patients without risk factors for MDR pneumonia. The rationale for recommending broader empiric coverage for patients without increased risk for MDR infection is not provided. ${ }^{1}$

What are the implications of these recommendations for Canadian practice? The 2008 AMMI Canada recommendations ${ }^{3}$ include empiric regimens with narrower spectrums of activity than those recommended by the 2016 IDSA/ATS guidelines. ${ }^{1}$ In fact, even in patients with hospital-acquired pneumonia who are regarded to be at increased risk of infection with an MDR pathogen but who have mild or moderate illness, the AMMI Canada guidelines list ceftriaxone and moxifloxacin monotherapy as suitable options. ${ }^{3}$ Empiric antipseudomonal coverage is required only for patients with severe illness (e.g., hypotension, organ dysfunction, hypoxia associated with need for mechanical ventilation) and in those with suspected Pseudomonas aeruginosa infection. ${ }^{3}$ Therefore, strict adherence to the 2016 IDSA/ATS recommendations could lead to more widespread use of broadspectrum antipseudomonal antibiotics in the Canadian population of patients with hospital-acquired or ventilator-associated pneumonia.

A shift in practice of this nature should compel us to ask whether following the IDSA/ATS recommendations will improve patient outcomes. Although there are no data specifically related to the 2016 IDSA/ATS guidelines, a 2011 study called into question the benefits of adhering to the previous edition of these guidelines. ${ }^{4}$ This prospective, observational study of intensive care patients at risk for MDR pneumonia examined whether outcomes were better among patients who received empiric therapy compliant with the 2005 IDSA/ATS guidelines than among those who received noncompliant therapy. ${ }^{4}$ The authors found that $89 \%$ of noncompliance was due to non-use of dual treatment for gram-negative pathogens. ${ }^{4}$ Guideline adherence was associated with higher 28-day mortality, regardless of the identified pathogen (adjusted hazard ratio 1.56, 95\% confidence interval $1.00-2.44){ }^{4}$ The authors proposed that this finding could reflect the excess harm versus benefit associated with combination gram-negative treatment. ${ }^{4}$ 
While far from definitive, this study suggests caution with regard to strict adherence to guidelines. This caution is echoed in the 2016 IDSA/ATS guidelines themselves, which include a new recommendation that each hospital regularly generate and disseminate a local antibiogram, ideally tailored to the intensive care population. ${ }^{1}$ This recommendation is significant in its acknowledgement that the prevalence and antimicrobial susceptibilities of MDR pathogens vary across wards and institutions, and that these should be the primary factors guiding empiric therapy.

The overarching goals of therapy for hospital-acquired and ventilator-associated pneumonia are improvement of patient outcomes and antimicrobial stewardship. The 2016 IDSA/ATS guidelines can serve as a useful tool, but we recommend a few key actions for pharmacists to take in their adoption: (1) develop relationships with the hospital's medical microbiology department to optimize communication of antibiograms, (2) incorporate local antibiograms into therapeutic decision-making at a population level, and (3) carefully consider the patient's clinical condition and risk factors for MDR pathogens when making patient-level decisions. Clinical judgment is paramount, and pharmacists can certainly be leaders in ensuring judicious treatment of hospitalacquired and ventilator-associated pneumonia.

\section{References}

1. Kalil AC, Metersky ML, Klompas M, Muscedere J, Sweeney DA, Palmer LB, et al. Management of adults with hospital-acquired and ventilator-associated pneumonia: 2016 clinical practice guidelines by the Infectious Diseases Society of America and the American Thoracic Society. Clin Infect Dis. 2016;63(5): e61-e111.
2. American Thoracic Society; Infectious Diseases Society of America. Guidelines for the management of adults with hospital-acquired, ventilator-associated, and healthcare-associated pneumonia. Am J Respir Crit Care Med. 2005;171(4): 388-416.

3. Rotstein C, Evans G, Born A, Grossman R, Light RB, Magder S, et al. Clinical practice guidelines for hospital-acquired pneumonia and ventilator-associated pneumonia in adults. Can J Infect Dis Med Microbiol. 2008;19(1):19-53.

4. Kett DH, Cano E, Quartin AA, Mangino JE, Zervos MJ, Peyrani P, et al.; Improving Medicine through Pathway Assessment of Critical Therapy of Hospital-Acquired Pneumonia (IMPACT-HAP) Investigators. Implementation of guidelines for management of possible multidrug-resistant pneumonia in intensive care: an observational, multicentre cohort study. Lancet Infect Dis. 2011;11(3):181-9.

Hilary Wu, BSc(Pharm), ACPR, PharmD

Providence Health Care

Vancouver, British Columbia

Curtis Harder, BSC(Pharm), ACPR, PharmD

Royal Jubilee Hospital, Island Health

Victoria, British Columbia

Celia Culley, BSP, ACPR, PharmD

Royal Jubilee Hospital, Island Health

Victoria, British Columbia

Competing interests: None declared. 